\title{
Study of Quench Protection for the Nb3Sn Low- $\beta$ Quadrupole for the LHC Luminosity Upgrade (HiLumi-LHC)
}

\author{
Ezio Todesco \\ CERN, Geneva, Switzerland \\ V. Marinozzi, G. Bellomo and M. Sorbi \\ University of Milano and INFN-LASA, Milan, Italy \\ G. Ambrosio and G. Chlachidze \\ Fermi National Accelerator Laboratory, Batavia, USA \\ H. Felice and M. Marchevsky \\ LBNL, Berkeley, USA \\ T. Salmi \\ Tampere University of Technology, Tampere, Finland
}

Keywords: Niobium compounds, quench protection, superconducting accelerators

\begin{abstract}
The HiLumi program is aiming to develop and build new Nb3Sn, high-field (12 T) and large aperture $(150 \mathrm{~mm})$ superconducting quadrupoles, which will be inserted in the LHC interaction regions and will provide the final focusing of the beam, in the program of the luminosity upgrade. The quench protection of these magnets is one of the most challenging aspects, mainly because of the large value of the magnet inductance $(160 \mathrm{mH}$ for the configuration with two $8 \mathrm{~m}$ long magnets in series), of the large value of the stored magnetic energy density in the coils $(0.12 \mathrm{~J} / \mathrm{mm} 3$, a factor 2 larger than in the conventional NbTi quadrupoles) and of the use of Nb3Sn as conductor, which has never been used for large accelerator magnets. Previous works have demonstrated that a "standard" conservative analysis, assuming quench heaters only on the coils outer layer, gives high hot spot temperature, close to the design limit (350 K). In this paper, a new study of quench protection is presented. The benefic effects of large $\mathrm{dI} / \mathrm{dt}$ during the discharge and other dynamic effects are discussed together with options for having a partial coverage of the inner layer by quench heaters. The analysis is validated by experimental data from R\&D Nb3Sn quadrupole magnets.
\end{abstract}

Presented at: ASC 2014, 10-15 August, Charlotte, USA

Geneva, Switzerland

June 2015 


\title{
Study of Quench Protection for the $\mathrm{Nb}_{3} \mathrm{Sn}$ Low- $\beta$ Quadrupole for the LHC Luminosity Upgrade (HiLumi-LHC)
}

\author{
Vittorio Marinozzi, Giorgio Ambrosio, Giovanni Bellomo, Guram Chlachidze, Helene Felice, \\ Maxim Marchevsky, Tiina Salmi, Massimo Sorbi, and Ezio Todesco
}

\begin{abstract}
The HiLumi program is aiming to develop and build new $\mathrm{Nb}_{3} \mathrm{Sn}$, high-field $(12 \mathrm{~T})$ and large aperture $(150$ mm) superconducting quadrupoles, which will be inserted in the LHC interaction regions and will provide the final focusing of the beam, in the program of the luminosity upgrade. The quench protection of these magnets is one of the most challenging aspects, mainly because of the large value of the magnet inductance $(160 \mathrm{mH}$ for the configuration with two $8 \mathrm{~m}$ long magnets in series), of the large value of the stored magnetic energy density in the coils $\left(0.12 \mathrm{~J} / \mathrm{mm}^{3}\right.$, a factor 2 larger than in the conventional NbTi quadrupoles) and of the use of $\mathrm{Nb}_{3} \mathrm{Sn}$ as conductor, which has never been used for large accelerator magnets. Previous works have demonstrated that a "standard" conservative analysis, assuming quench heaters only on the coils outer layer, gives high hot spot temperature, close to the design limit (350 K). In this paper, a new study of quench protection is presented. The benefic effects of large $d I / d t$ during the discharge and other dynamic effects are discussed together with options for having a partial coverage of the inner layer by quench heaters. The analysis is validated by experimental data from $R \& D N_{3} S n$ quadrupole magnets.
\end{abstract}

Index Terms - Niobium compounds, quench protection, superconducting accelerators.

\section{INTRODUCTION}

$\mathrm{T}$ HE Large Hadron Collider (LHC) at CERN has produced proton-proton collisions up to $8 \mathrm{TeV}$ centreof-mass energy in the period 2010-2013. After the commissioning towards $14 \mathrm{TeV}$ in 2015 , and towards a designed luminosity of $1-2 \times 10^{34} \mathrm{~cm}^{-2} \mathrm{~s}^{-1}$, it is expected to reach $300 \mathrm{fb}^{-1}$ at the horizon of 2021. Running the accelerator after this date without a significant luminosity increase will lead only to marginal statistical gain; therefore a luminosity upgrade, named High Luminosity LHC (HLLHC), has been planned, with the target of accumulating $3000 \mathrm{fb}^{-1}$ in the successive 10 years. This can be achieved with a more intense beam and by using superconducting magnets with better performance than the present $\mathrm{Nb}-\mathrm{Ti}$,

V. Marinozzi, G. Bellomo and M. Sorbi are with University of Milano and INFN-LASA, Milan, Italy, email: vittorio.marinozzi@mi,infn,it.

G. Ambrosio and G. Chlachidze are with Fermi National Accelerator Laboratory, Batavia, IL 60510-5011, USA, email: giorgioa@fnal.gov.

H. Felice and M. Marchevsky are with Lawrence Berkeley National Laboratory, Berkeley, CA 94720, USA, email: mmartchevskii@lbl.gov.

T. Salmi is with Tampere University of Technology, Tampere, Finland, email: tiina.salmi@tut.fi.

E. Todesco is with CERN, TE-Dep, Geneve, Switzerland, email: ezio.todesco@cern.ch.

The research leading to these results has received funding from the European Commission under the FP7 project HiLumi LHC, GA no. 284404, co-funded by the DoE, USA and KEK, Japan. specifically replacing the low- $\beta$ triplet quadrupole magnets with larger aperture magnets. The selected quadrupole design, called MQXF, presents a very large aperture (150 $\mathrm{mm}$ in magnet bore diameter) and a peak field on the conductor of $12.2 \mathrm{~T}$, thanks to a $\mathrm{Nb}_{3} \mathrm{Sn}$ conductor. Table I lists the main parameters of the magnet, and Fig. 1 represents the cross section of the magnet in the $1^{\text {st }}$ quadrant.

Because of the high magnetic field and of the large magnetic energy density in the coils (about $0.12 \mathrm{~J} / \mathrm{mm}^{3}$, compared to $0.05-0.06 \mathrm{~J} / \mathrm{mm}^{3}$ in the current $\mathrm{Nb}-\mathrm{Ti}$ low- $\beta$ triplet magnets), the quench protection is one of the most challenging aspects of these magnets, requiring a detailed study to verify the safe working conditions [1].

TABLE I MaIN PARAMETERS OF MQXF

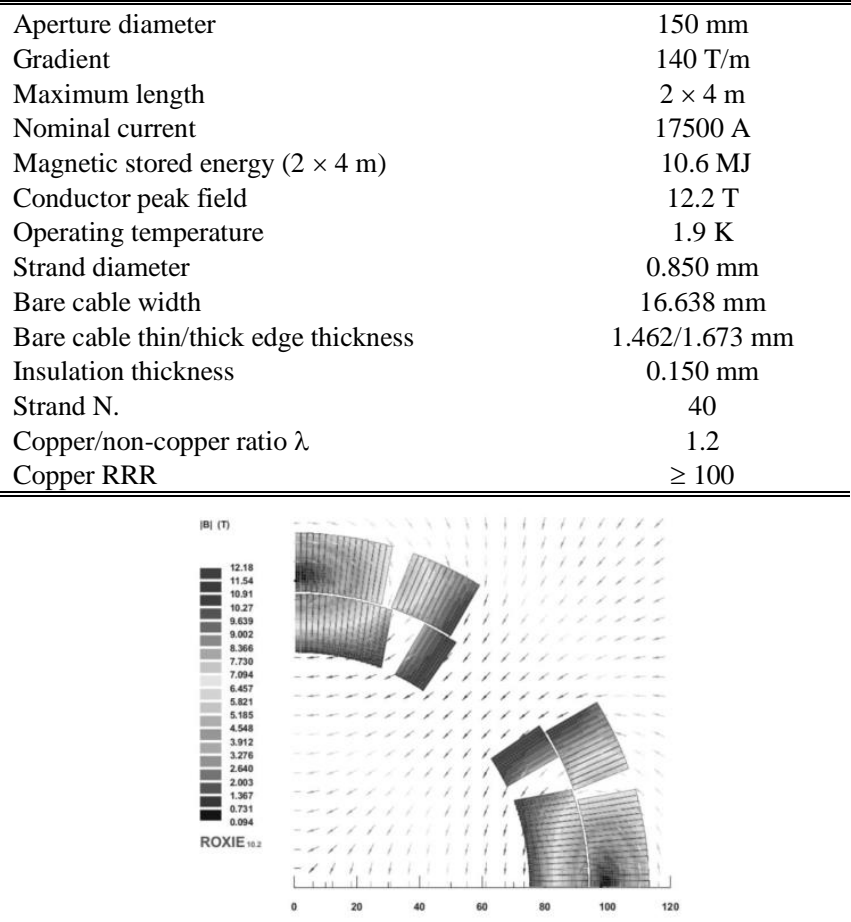

Fig. 1. One quadrant cross section of MQXF with magnetic field map.

In a previous paper [2] the MQXF quench protection was analysed with two different codes, and the conclusion was that the minimal target to keep the peak temperature below $350 \mathrm{~K}$ was hardly achieved. This was not leaving margin for any uncertainties, such as material properties or additional temporal delays in the protection activation. Further studies on the quench protection have been done to explore the presence of hidden margins. One of the most important was the possible gain due to the reduced value of the apparent inductance, probably caused by rapid $d I / d t$ effects; this lower inductance was experimentally observed 
during the test of HQ magnets with fast current decays, which was more rapid $(\sim 30 \%)$ than in the simulations. In this paper we discuss some models to explain the faster current decay that has been experimentally measured. These models are used to update the simulation scenario for MQXF, where also inner layer quench heaters are considered.

\section{COUPling With Metallic COMPONENTS}

A first explanation of the apparent inductance decrease during a fast decay has been envisaged by electromagnetic coupling of the coils with the metallic parts of the magnet, such as the collar or the shell: during the discharge, because of the high $d I / d t$, eddy currents flow in these metallic parts and extract part of the stored energy.

As a first approximation, the collar or the shell can be represented as an infinitely long external thin cylinder. In this cylinder, assuming that the main coils are a perfect $\cos 2 \vartheta$ magnet, $\cos 2 \vartheta$ eddy currents flow during the decay, with an intrinsic time constant $\tau$ [3]. As a consequence, the coupling of the cylinder with the quadrupole is equivalent to the coupling of a primary circuit (the circuit of the coils) with a secondary circuit (the circuit of cylinder with equivalent current $I_{2}$, inductance $L_{2}$ and resistance $R_{2}$ ), with mutual inductance $M$. The equivalence between magnetic-energy/power-dissipated in the cylinder and magnetic-energy/power-dissipated in the secondary circuit gives the following values for the electrical parameter of the secondary circuit [4]:

$R_{2}=\frac{\pi}{4} \frac{\varrho}{a d}$

$L_{2}=\frac{\pi}{16} \mu_{0}$

$M=\frac{\pi}{32} \mu_{0} \frac{c_{2}{ }^{2}+{c_{1}}^{2}}{a^{2}} N_{1}$

where $\varrho$ is the cylinder resistivity, $a$ is the cylinder radius, $d$ is the cylinder thickness, $c_{2}$ and $c_{1}$ are respectively the outer and inner radius of the main coils, and $N_{1}$ is the total number of the main turns.

The solution of the equation of the current in the coil coupled with the equivalent circuit gives a current decrease faster than a pure exponential.

\section{DYNAMIC INDUCTANCE FOR INTER-FILAMENT COUPLING CURRENTS}

Under changes of the external field, the superconducting strands are affected by Inter-Filament Coupling Currents (IFCC). During the quench process, the field variation is so large that the field flux produced by these currents could not be negligible, therefore it should be taken into account in the calculation of the magnet differential inductance during the current discharge.

The total field $B_{i}$ on the strand follows the equation [5]: $B_{i}=B_{e}-\dot{B}_{i} \tau$

where $B_{e}$ is the external field, and $\tau$ the IFCC decay time constant [5]. Even if the copper resistivity is not constant (in space and in time) because of the magneto-resistivity, $\tau$ has been considered constant and corresponding to an average field value. Assuming that $B_{e}$ decreases exponentially, the solution of (4) is

$B_{i}=\frac{B_{0}}{\tau-\tau_{e}}\left(\tau e^{-\frac{t}{\tau}}-\tau_{e} e^{-\frac{t}{\tau_{e}}}\right)$ where $\tau_{e}$ is the external field time constant and $B_{0}$ the field at $t=0$. The IFCC produce a magnetization $M$ :

$M=2 \lambda \tau \frac{\dot{B}_{i}}{\mu_{0}}$

which can be written also as

$M=\frac{\chi}{1+\chi} \frac{B_{i}}{\mu_{0}}$

where $\chi$ is the magnetic susceptivity and $\lambda<1$ is the product of the metal fraction in the strand and the conductor packing factor. Combining equations (5), (6) and (7), the susceptivity $\chi$ related to the IFCC can be obtained:

$\chi=\frac{2 \lambda \tau\left(e^{-\frac{t}{\tau_{e}}}-e^{-\frac{t}{\tau}}\right)}{\tau e^{-\frac{t}{\tau}}-\tau_{e} e^{-\frac{t}{\tau_{e}}}-2 \lambda \tau\left(e^{-\frac{t}{\tau_{e}}}-e^{-\frac{t}{\tau}}\right)}$

The dynamic inductance, defined as:

$L_{d}=\frac{d \phi}{d I}=\frac{1}{I} \frac{d U}{d I}$

can be found through the expression of the stored energy variation per unit volume $d u$ :

$d u=H d B=\mu_{0} H d H+\mu_{0} \chi H d H+\mu_{0} H^{2} \frac{d \chi}{d I}$

The dynamic inductance variation due to the IFCC can be found integrating over the coils volume the last two terms in (10), and substituting the result in (9)

$L_{d}=\int\left\{(1+\chi) \frac{\mu_{0}}{2 I} \frac{d H^{2}}{d I}+\frac{\mu_{0} H^{2}}{I} \frac{d \chi}{d I}\right\} d V$

The first term in (10), integrated over the whole space, gives the static inductance, assuming that the magnetization does not affect the magnetic field $H$.

\section{DYNAMIC INDUCTANCE EXPERIMENTAL VALIDATION}

It has been experimentally observed that during a quench the current decreases faster than expected [6], [7], already at the very beginning of the discharge.

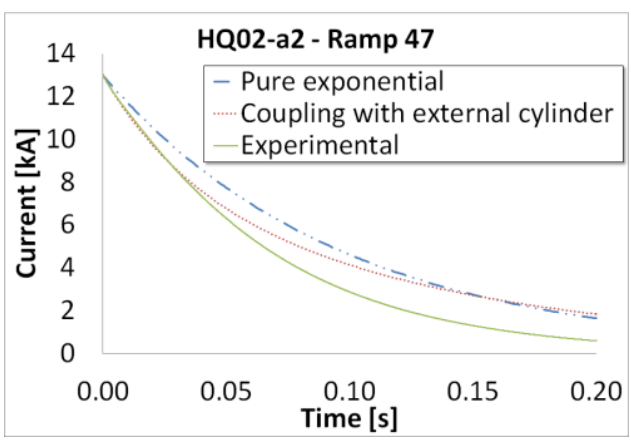

Fig. 2. Comparison between an experimental current decay from HQ02 (solid green), a pure exponential (dashed blue) and a current decay obtained considering the coupling with an external cylinder (pointed brown).

The experimental data in Fig. 2 are from HQ02 [8], a $\mathrm{Nb}_{3} \mathrm{Sn}$ quadrupole magnet, with an external dump resistor $R_{d}$ of $60 \mathrm{~m} \Omega$, starting from $13 \mathrm{kA}(70 \%$ of the shortsample-limit at $1.9 \mathrm{~K})$. Considering the nonlinear inductance of $5.8 \mathrm{mH}$ at $13 \mathrm{kA}$, one gets an exponential decay (blue curve, with a time constant of $97 \mathrm{~ms}$ ). On the other hand, the experimental curve has an initial time constant about $30 \%$ smaller; this cannot be explained as a quench back since it happens at the very beginning of the decay, whereas the quench back needs some time to act.

In Fig. 2 the result of the model described in section II (coupling with the external cylinder) is shown, considering 
the following values: $R_{2}=4.5 \mu \Omega, L_{2}=0.2 \mu \mathrm{H}, \quad M=$ $19.3 \mu \mathrm{H}$. for the resistance calculation, the nominal aluminium resistivity at $2 \mathrm{~K}$ has been used, from the MATPRO database [9]. Actually, the magnet collar is laminated, therefore an effective, higher resistivity could be used in order to take into account the surface resistance of the laminations; for lacking of reliable data, nominal value of bulk material has been used. The obtained current decay fits well at the beginning (from $t=0$ to $t=30 \mathrm{~ms}$ ), but later the current decays again much slower than the experimental curve.

For the model described in section III (inductance variation for the IFCC), an extension to the quench simulation code QLASA [10] has been implemented, and the results have been compared with the experimental data in Fig. 3. In the code at each time step the inductance is recalculated, assuming (8) for the permittivity in the coils, and a $\cos 2 \vartheta$ magnetic field for the volume integration (11).

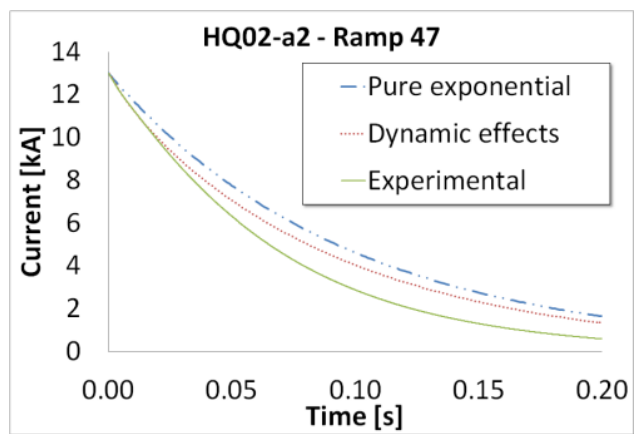

Fig. 3. Comparison between an experimental current decay from HQ02 (solid green), a pure exponential (dashed blue) and a simulation made considering the dynamic effects described in section III (pointed red).

The simulation [11] has been performed considering $\lambda=$ 0.7 and $\tau=15 \mathrm{~ms}$ [5]. Also in this case, the simulation which considers the IFCC fits the experimental one at the beginning, until about $10 \mathrm{~ms}$; then, the experimental curve decays faster. However at this time a quench back can be supposed to occur: in Fig. 4 the power per unit volume dissipated by the IFCC $\left(p=\frac{2 \dot{B}^{2} \tau}{\mu_{0}}\right)$ is represented and compared to the joule effect dissipation $\left(p=\varrho J^{2}\right)$. The presence of fast quench back starting after about 5-10 ms has been also confirmed by the results of the CLIQ [12] tests performed on the same magnet at CERN.

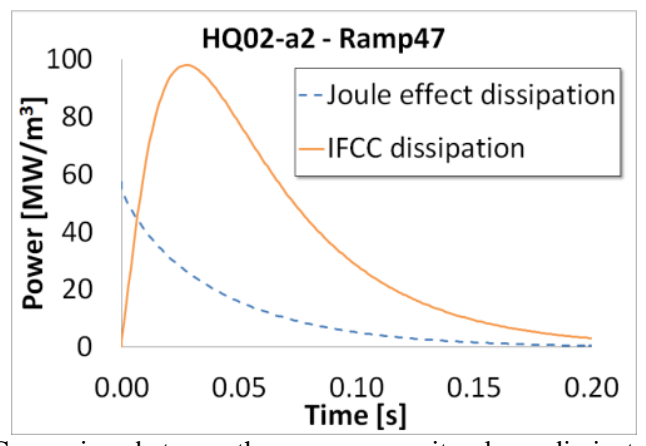

Fig. 4. Comparison between the power per unit volume dissipated by the eddy currents (solid orange) and the power dissipated by the joule effect during a quench in a high field zone ( $8 \mathrm{~T}$ ) (dashed blue)

A quench back after $10 \mathrm{~ms}$ and the additional power due to the IFCC have been introduced in the model, in order to fit the curve; quench back is modelled as a widespread quench in the whole magnet after $10 \mathrm{~ms}$. The simulation is now almost coincident with the experimental curve, as represented in Fig. 5. A curve which considers only the dynamic effects is also shown, in order to point out that the quench back (with the additional power dissipation for IFCC) affects the decay more than dynamic effects.

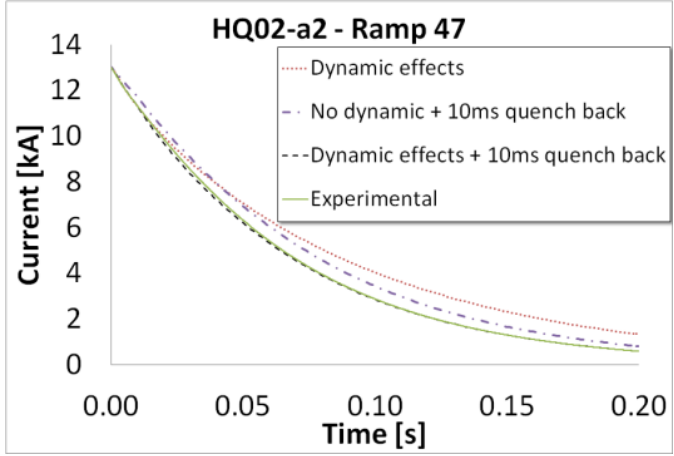

Fig. 5. Comparison between an experimental current decay from HQ02 (solid green), a simulation made considering the dynamic effects (pointed red), one considering dynamic effects plus quench back after $10 \mathrm{~ms}$ (dashed black) and one considering quench back after $10 \mathrm{~ms}$, but no dynamic effects (point-dashed purple).

On the other hand the $10 \mathrm{~ms}$ quench back alone cannot justify such a sudden change of the current slope at the very beginning of the discharge, as shown in Fig. 5, where the point-dashed purple curve represents a current decay with $10 \mathrm{~ms}$ quench back at the magnet nominal inductance.

The computed dynamic inductance during the discharge, according to the model of the IFCC described in section III, is shown in Fig. 6, and it is compared to the HQ nominal inductance (measured at low $d I / d t$ [13], a high current value is used for reference).

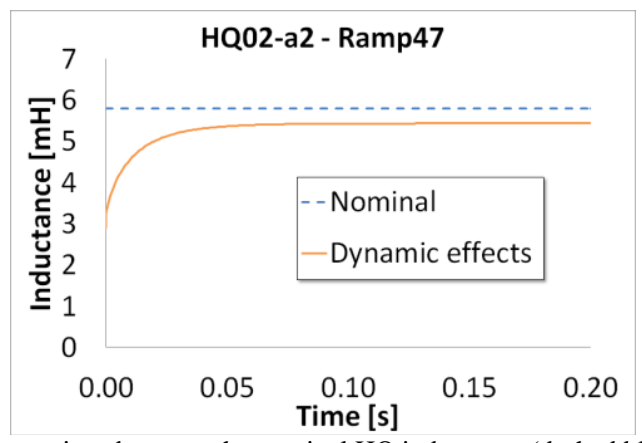

Fig. 6. Comparison between the nominal HQ inductance (dashed blue) and the differential inductance obtained considering the dynamic effects described in section II (solid orange).

It can be noted that the dynamic effects appreciably affect the curve at the beginning of the decay, when $t \lesssim \tau$, where the dynamic inductance is $\sim 30 \%$ lower than the nominal one. Then the dynamic inductance saturates, because the IFCC decay within 2-3 $\tau$. Note that the dynamic inductance saturates to a value slightly lower (about $1 \%$ ) than the magnet nominal inductance: this is due to the fact that the magnetization due to IFCC continues to change since for large values of $t$ the susceptivity given in (8) saturates to a not null, negative value, therefore in (11) there is always a small reduction.

In conclusion, this study shows that faster current decays during a discharge can be predicted by inductance variation due to the IFCC, as shown in section III; this model is also consistent with a very rapid quench back, because of the additional power dissipated in the conductor. Instead, the variation of inductance due to coupled current in metallic components, as in the model described in section II, allows to fit experimental data at the very beginning of current decay, but it cannot justify a fast quench back in the coils. 


\section{MQXF QUENCH HEATERS}

MQXF protection is relying on quench heaters on the outer layer [2], [14]. A possible way to improve protection is to add protection heaters also on the inner layer (IL). Various designs for the IL protection heaters have been proposed and are here summarized

A. Stainless steel-only design

This layout comes from the earlier design used in Long Quadrupoles (LQ) [15]. It involves regularly spaced heating stations separated by wider bridges (Fig. 7 up-left). The material is uniform stainless steel (SS304) with thickness $d=25 \mu \mathrm{m}$.

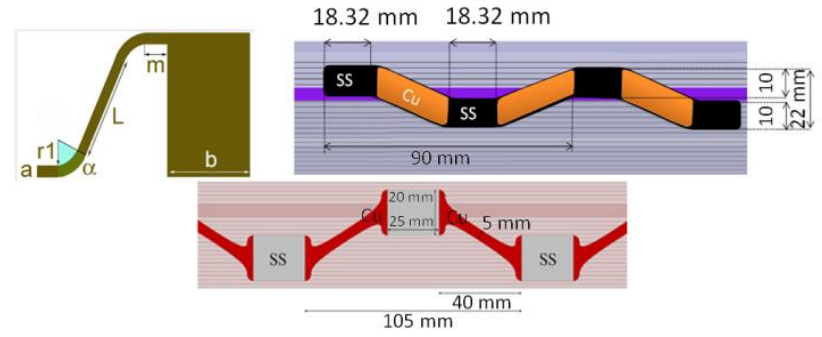

Fig. 7. Top-Left: a sketch showing a period of heating station stainless steel-only design. Top-Right: IL heater layout for copper-plated heater design 1. Bottom: IL heater layout for copper-plated heater design 2.

\section{B. Copper-plated heater designs}

The copper plating allows to better protect a long magnet w.r.t the heating stations technique: in fact, the resistance of the copper part is negligible and heating stations can be made longer without exceeding the maximum voltage. The design 1 is shown in Fig. 7 top-right. In the design 2 the copper-plated bridges are made narrower with respect to the design 1, to allow further space for cooling the coils and also to allow more space for perforation, avoiding the formation of helium bubbles that have been observed in the HQ program. The distance between heating stations is in both cases of the order of $100 \mathrm{~mm}$; having a longitudinal propagation speed of $20 \mathrm{~m} / \mathrm{s}$ [13], the locations between heating stations get quenched $2.5 \mathrm{~ms}$ later, which is considered as an acceptable delay. Terminals of the heating stations are copper-plated to improve current flow uniformity. The design is shown in Fig. 7, bottom.

\section{MQXF Hot SPOT TEMPERATURE}

The MQXF hot spot temperature has been calculated considering also the presence of protection heaters $(\mathrm{PH})$ on the IL (see section V) and the dynamic effects described in section III. The results show that the three IL heater designs are almost equivalent for the hot spot temperature calculation (the hot spot temperature changes are about \pm 5 $\mathrm{K}$ in the three designs). The $\mathrm{PH}$ delay time have been obtained using CoHDA [16]: the heaters-induced quench is assumed to occur at the same time in all the turns which are in direct thermal contact with the strip. The delay times shown in Table II are therefore averages. More details on the PH simulation using QLASA can be found in [2] and [11]. The simulations have been performed considering the case of two $8 \mathrm{~m}$ long magnets in series, according to the nominal protection parameters, which are reported in Table II. Table III reports hot spot temperature for four different cases, with/without IL heaters and with/without dynamic effects. The hot spot temperature calculation has been performed considering material properties from the MATPRO database [9].
TABLE II MQXF PROTECTION PARAMETERS

\begin{tabular}{lc}
\hline \hline Dump resistor (maximum voltage to ground) & $46 \mathrm{~m} \Omega(800 \mathrm{~V})$ \\
Voltage threshold & $100 \mathrm{mV}$ \\
Validation time & $10 \mathrm{~ms}$ \\
Heaters delay time from firing (inner-layer) & $12 \mathrm{~ms}$ \\
Heaters delay time from firing (outer-layer) & $16 \mathrm{~ms}$ \\
\hline \hline
\end{tabular}

The insertion of PH on the IL causes a decrease of $\sim 40 \mathrm{~K}$ of the hot spot temperature; the dynamic effects give an additional $\sim 25 \mathrm{~K}$ decrease. Although the dynamic effects are here less effective than in the previously examined HQ case, because in MQXF the initial $d I / d t$ is lower $(\sim 10 \mathrm{kA} / \mathrm{s}$ vs. $\sim 100 \mathrm{kA} / \mathrm{s}$ ), they appreciably affect the current decay, reducing the MIITs developed during the discharge and lowering the final magnet hot spot temperature. In conclusion, considering the dynamic effects on the inductance, magnet safety is ensured in both cases with or without IL PH.

\section{TABLE III MQXF MIITS/HOT SPOT TEMPERATURE}

\begin{tabular}{cccc}
\hline \hline No IL-PH & IL-PH & No IL-PH & IL-PH \\
no dynamic & no dynamic & dynamic & dynamic \\
\hline $35.5 \mathrm{MA}^{2} \mathrm{~s}$ & $32.8 \mathrm{MA}^{2} \mathrm{~s}$ & $39.9 \mathrm{MA}^{2} \mathrm{~s}$ & $31.1 \mathrm{MA}^{2} \mathrm{~s}$ \\
$330 \mathrm{~K}$ & $290 \mathrm{~K}$ & $306 \mathrm{~K}$ & $266 \mathrm{~K}$ \\
\hline \hline
\end{tabular}

\section{MQXF PROTECTION REDUNDANCY}

Simulations have been repeated for the four cases previously examined, considering a failure of half of the $\mathrm{PH}$. The results are reported in Table IV.

TABLE IV MIITS/HOT SPOT TEMPERATURE WITH HALF HEATERS

\begin{tabular}{cccc}
\hline \hline No IL-PH & IL-PH & No IL-PH & IL-PH \\
no dynamic & no dynamic & dynamic & dynamic \\
\hline $37.8 \mathrm{MA}^{2} \mathrm{~s}$ & $34.2 \mathrm{MA}^{2} \mathrm{~s}$ & $36.5 \mathrm{MA}^{2} \mathrm{~s}$ & $32.9 \mathrm{MA}^{2} \mathrm{~s}$ \\
$365 \mathrm{~K}$ & $311 \mathrm{~K}$ & $341 \mathrm{~K}$ & $287 \mathrm{~K}$ \\
\hline \hline
\end{tabular}

There is an increase of the hot spot temperature of $\sim 20 \mathrm{~K}$ in the IL-PH case, and of $\sim 35 \mathrm{~K}$ in the no IL-PH case, respect to Table III. According to this model, considering also the dynamic effects, redundancy is provided only if inner layer protection heaters are inserted.

\section{CONCLUSIONS}

Previous works on the MQXF quench protection showed that the hot spot temperature was too close to the design limit of $350 \mathrm{~K}$ [2]. On the other hand, experimental evidence of dynamic effects for the HQ quadrupole showed a faster current decay. Here we study these effects, developing two models described in sections II and III. The IFCC model described in section III allows to reproduce the observed faster discharge, and the additional power dissipation in the conductor explains rapid quench back. The model has been applied for the MQXF simulations in order to calculate the dynamic inductance during the discharge, according to the nominal protection parameters.

Additionally, quench heaters in the IL have been designed and implemented in the simulations. A faster discharge can be clearly observed, therefore less MIITs are produced and the hot spot temperature is now reasonably lower than the design limit of $350 \mathrm{~K}$; therefore, under these assumptions, the magnet can be considered safe also without IL quench heaters. Anyway, redundancy is ensured in case of half heaters failure, provided that IL protection heaters are inserted. Further improvement to the MQXF protection supposed to come from quench back has not been considered in this work. 
This is the author's version of an article that has been published in this journal. Changes were made to this version by the publisher prior to publication.

The final version of record is available at http://dx.doi.org/10.1109/TASC.2014.2383435

\section{REFERENCES}

[1] E. Todesco, "Quench limits in the next generation of magnets" CERN Yellow Report 2013-006 10-16 (2013).

[2] G. Manfreda, G. Ambrosio, V. Marinozzi, T. Salmi, M. Sorbi, G. Volpini, "Quench Protection Study of the $\mathrm{Nb}_{3} \mathrm{Sn}$ Low- $\beta$ Quadrupole for the LHC Luminosity Upgrade," IEEE Trans. on Appl. Supercond., vol. 24, no. 3, June 2014, Art. No. 4700405.

[3] W. R. Smythe, "Static and Dynamic Electricity", McGraw-Hill, 1950, p.411

[4] M. Sorbi and G. Bellomo, "The role of eddy currents during a quench in quadrupole superconducting magnets", internal note, https://lhc-div-mms.web.cern.ch/lhc-div-

mms/tests/MAG/docum/hilumi/Presentations/2013-09-

23_ms_eddy.docx

[5] M.N. Wilson, "Superconducting Magnets", chapter 8, pp. 176-180, Clarendon Press Oxford, 1983.

[6] V. Marinozzi, "Study of the quench propagation in the high-field superconducting quadrupoles for the LHC luminosity upgrade,' Master's thesis, University of Milan, 2013, Tech. Rep. TD-13-013, Fermilab Technical Division, 2013. Presented on 23 July 2013.

[7] L. Rossi, "Study of superconducting-to-resistive transition for the USLARP, large high field quadrupoles for the LHC upgrade," Tech. Rep. TD-12-11, Fermilab Technical Division, 2012.

[8] H. Felice et al., "Design of HQ - a high field large bore Nb3Sn quadrupole magnet for LARP," IEEE Trans. on Appl. Supercond. vol. 19 , no. 3, pp. 1235-1239, 2009.

[9] G. Manfreda, L. Rossi, and M. Sorbi, "MATPRO upgraded version 2012: a computer library of material property at cryogenic temperature," Tech. Rep. INFN-12-04/MI, INFN, April 2012.
[10] L. Rossi and M. Sorbi, "QLASA: A computer code for quench simulation in adiabatic multicoil superconducting windings," Nat. Inst. of Nucl. Phys. (INFN), Rome, Italy, Tech. Rep. TC-04-13,2004.

[11] V. Marinozzi, "Guidelines for the quench analysis of $\mathrm{Nb3Sn}$ accelerator magnets using QLASA," Tech. Rep. TD-13-008, Fermilab Technical Division, 2013.

[12] E. Ravaioli, V. I. Datskov, G. Kirby, H. H. J. Ten Kate and A. P. Verweij, "A new hybrid protection system for high-field superconducting magnets”, Supercond. Sci. Technol., vol. 27, no. 4, 044023, 2014.

[13] H. Bajas et al., "Cold Test Results of the LARP HQ Nb3Sn quadrupole magnet at $1.9 \mathrm{~K}$ ", IEEE Trans. on Appl. Supercond., vol 23, no. 3, 2013, Art. No. 4002606.

[14] T. Salmi, G. Ambrosio, S. Caspi, G. Chlachidze, H. Felice, M. Marchevsky, S. Prestemon, H. H. J. ten Kate, "Protection heater delay time optimization in high-field Nb3Sn magnets", IEEE Trans. on Appl. Supercond., vol. 24, no. 3, 2013, Art. No. 4701305.

[15] G. Ambrosio et al., "Test results of the first $3.7 \mathrm{~m}$ long Nb3Sn quadrupole by LARP and future plans," IEEE Trans. on Appl. Supercond., vol. 21, no. 3, pp. 1858-1862, 2011.

[16] T. Salmi, D. Arbelaez, S. Caspi, H. Felice, M. G. T. Mentink, S. Prestemon, A. Stenvall, and H. H. J. ten Kate, "A Novel Computer Code for Modeling Quench Protection Heaters in High-Field Nb3Sn Accelerator Magnets", IEEE Trans. on Appl. Supercond., vol. 24, no. 4, 2014, Art. No. 4701810. 